\title{
Clear Lens Extraction in Primary Angle-closure Disease-Pros and Cons
}

\author{
Syed Shoeb Ahmad, ${ }^{1}$ Ghuncha Khatoon, ${ }^{2}$ Iram Jahan, ${ }^{2}$ Md Naeem Arafat, ${ }^{2}$ Anwar Husain, ${ }^{2}$ and Tooba Hayat ${ }^{2}$
}

1. Ibn Sina Academy of Medieval Medicine and Sciences, Aligarh, India; 2. Ajmal Khan Tibbiya College, Aligarh, India

DOI: https://doi.org/10.17925/USOR.2020.13.1.23

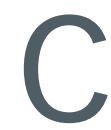
ataract extraction for management of primary angle-closure disease has been successfully practiced for many years. However, the extraction of a clear lens for glaucoma management, when visual symptoms are minimal, is a controversial subject. A few studies have reported on the outcomes of clear lens extraction without resorting to conventional methods, such as drugs and lasers. Others have compared clear lens extraction with trabeculectomy or iridotomy. Most have reported successful outcomes with this surgical modality, in terms of intraocular pressure control, reduction in the number of glaucoma medications, opening of the anterior chamber angle, visual gain, and improvement in quality of life. Unfortunately, the studies on clear lens extraction are hampered by both positive and negative aspects in their execution. This review was undertaken to debate the points presented in the studies in order to have a better understanding of the advantages and disadvantages of clear lens extraction in primary angle-closure disease.

\section{Keywords}

Lens, crystalline, glaucoma, angle closure, phacoemulsification, cataract

Disclosures: Syed Shoeb Ahmad, Ghuncha Khatoon, Iram Jahan, Md Naeem Arafat, Anwar Husain and Tooba Hayat have no financial or non-financial relationships or activities to declare in relation to this article.

Review Process: Double-blind peer review.

Compliance with Ethics: This study involves a review of the literature and did not involve any studies with human or animal subjects performed by any of the authors.

Authorship: The named authors meet the International Committee of Medical Journal Editors (ICMJE) criteria for authorship of this manuscript, take responsibility for the integrity of the work as a whole, and have given final approval for the version to be published.

Access: This article is freely accessible at

touchOPHTHALMOLOGY.COM

(C) Touch Medical Media 2020

Received: March 5, 2020

Accepted: April 9, 2020

Published Online: May 11, 2020

Citation: US Ophthalmic Review. 2020;13(1):23-9

Corresponding Author: Syed Shoeb Ahmad, Ibn Sina Academy of Medieval Medicine and Sciences, Aligarh 202002, Uttar Pradesh, India.

E: syedshoebahmad@yahoo.com

Support: No funding was received in

the publication of this article.
Recently, new surgical modalities for management of primary angle-closure disease (PACD) have been proposed; the most controversial of which being clear lens extraction (CLE). The Effectiveness in Angle-closure Glaucoma of Lens Extraction (EAGLE) and other studies recommend CLE as the primary procedure of choice to manage eyes with PACD. ${ }^{1-5}$ However, the rationale for CLE without resorting to conventional management techniques has become a matter of debate. This review looks into the positive and negative aspects of the various studies performed regarding CLE and how they will mold our future discourse on this subject.

Several risk factors are associated with the development of PACD. The prevalence of primary angle-closure (PAC) is found to increase with age, which is attributable to the increasing bulk of the aging lens ${ }^{6.7}$ Progressive growth of the lens leads to a shallowing of approximately $0.35-0.50 \mathrm{~mm}$ in the anterior chamber depth by 50 years of age. ${ }^{8}$ Poley et al. have proposed intraocular pressure (IOP) elevation secondary to age-related lens expansion as a type of "phacomorphic ocular hypertension". The mean anterior chamber depth in eyes with PAC is around $1.8 \mathrm{~mm}$, which is $1.0 \mathrm{~mm}$ less than normal eyes. ${ }^{10}$ Eyes with PACD also have occludable angles; this is described as a "pre-glaucomatous stage" in the disease course. ${ }^{11}$ Gender is also a risk factor for PACD, with females being more susceptible due to crowding of structures in the anterior segment leading to decreased aqueous outflow facility. ${ }^{10}$ Other anatomical risk factors, apart from smaller anterior chamber width, area, and volume, are thicker iris, greater iris curvature and greater lens vault. ${ }^{12-14}$ Lens vault is defined as the part of the lens situated anteriorly to a plane drawn across the scleral spurs. It is recognized as an important factor in the development of acute angle-closure (AAC)..$^{15}$

The preceding discussion highlights the major role played by the lens in the pathogenesis of this condition. This has led to a widening acceptance of lens-based surgery to manage PACD. While cataract extraction in PACD is widely practiced, the role of CLE is still unresolved. Supporters and opponents of this procedure have both published their findings. However, CLE remains controversial in the absence of concrete evidence from either side. ${ }^{6}$ Hopefully, further studies will be conducted in the future to clarify the role of this procedure in our glaucoma management practices.

\section{Positive aspects of studies on clear lens extraction Historical evidence}

Before analyzing the studies on CLE, it would be interesting to review the historical evidence of cataract surgery in the management of PACD. In 1945, Guyton was probably the first to suggest cataract extraction as a means to control IOP in patients with primary angle-closure glaucoma (PACG). ${ }^{16}$ Subsequently, in 1971, Bigger and Becker demonstrated the lowering of IOP in glaucoma patients following cataract extraction. ${ }^{17}$ Civerchia and Balent performed extra-capsular cataract 
extraction in AAC with a successful reduction of IOP.18 Similarly, good IOP control after extra-capsular cataract extraction was demonstrated by Wishart and Atkinson in chronic PACG patients. ${ }^{19}$

Guning and Greve, in their study to compare extra-capsular cataract extraction with glaucoma filtering surgery in eyes with PACG, found better results with cataract extraction. ${ }^{20}$ Hata et al., in their study of primary phacoemulsification in PACG, compared cataract extraction with peripheral iridotomy and found the former technique to be superior in terms of IOP control and reduction in glaucoma medications. ${ }^{21}$ Similar results were reported when Jacobi et al. compared phacoemulsification with surgical iridectomy in patients with $A A C$; yielding overall better results in the group which underwent cataract extraction. The number of patients who subsequently underwent glaucoma surgery was five in the phacoemulsification group versus nine in the iridectomy group. ${ }^{22}$ Tham et al. compared phacoemulsification with phacotrabeculectomy and reported marginally better control of IOP in the phacotrabeculectomy group, but noted that phacotrabeculectomy was associated with more surgical complications (26.2\% versus $8.1 \%$ for phacotrabeculectomy and phacoemulsification, respectively). ${ }^{23}$ In a review of phacoemulsification in patients with glaucoma, 12 studies on chronic PACG showed the procedure was successful in reducing IOP by $30 \%$ and glaucoma medications by $58 \%$. The review also reported four studies on acute PACG that showed an average $71 \%$ reduction from presenting IOP. ${ }^{24}$

Cataract extraction for PACD has been practiced for many years with seemingly effective results. Such spectacular outcomes are also expected with CLE. However, due to the absence of visual symptoms in patients with clear lenses it becomes controversial whether to opt for CLE as a primary procedure and abandon the pharmacological and laser treatments used conventionally.

\section{Studies on clear lens extraction}

There are only a handful of reported studies regarding the effectiveness of CLE in clinical practice. However, Walland et al. have noted the appearance of such reports in non-peer-reviewed trade journals, indicating that it is being practiced on a wider scale than has been published. ${ }^{25}$ The EAGLE study was probably one of the first to assess the role of CLE in PAC/PACG. The study protocol was presented in 2011, and a large multicenter trial involving 30 hospitals in five countries was initiated. The inclusion criteria for the study were: individuals $\geq 50$ years of age with clear lenses and recently diagnosed PAC with $\mathrm{IOP} \geq 30 \mathrm{mmHg}$ or PACG. The study enrolled 419 individuals (PCA $n=155 ;$ PACG $n=263$ ). A web-based application randomized the participants into two arms: one group ( $n=208)$ underwent CLE and the other group $(n=211)$ received the standard practice of peripheral iridotomy and medications. ${ }^{1,26}$

Before the EAGLE study group could report their results, a few other studies on CLE published their findings. Tham and colleagues studied CLE versus trabeculectomy in cases of medically uncontrolled PACG. In their randomized study, 26 eyes underwent phacoemulsification, while 24 eyes had trabeculectomy. After 24 months of follow-up, IOP reduction was $8.4 \mathrm{mmHg}(34 \%)$ in the phacoemulsification group and $8.9 \mathrm{mmHg}$ (36\%) in the trabeculectomy group $(p=0.76) .^{2}$ Trabeculectomy-treated eyes required an average of 1.1 fewer drugs in comparison with phacoemulsification-treated eyes $(p<0.001)$. However, significantly more surgical complications were reported in the trabeculectomy-treated eyes
Table 1: Complications reported in trabeculectomy-operated eyes

\begin{tabular}{|l|l|}
\hline Complication & Eyes \\
\hline Cataract & 8 eyes \\
\hline $\begin{array}{l}\text { Over-drainage with mild choroidal detachment that resolved with } \\
\text { conservative measures }\end{array}$ & 2 eyes \\
\hline $\begin{array}{l}\text { Conjunctival wound leak that resolved with aqueous suppression } \\
\text { and eye patching }\end{array}$ & 2 eyes \\
\hline $\begin{array}{l}\text { Over-drainage requiring AC reformation and subsequent revision } \\
\text { of trabeculectomy }\end{array}$ & 1 eye \\
\hline
\end{tabular}

$A C=$ anterior chamber.

than in phacoemulsification-treated eyes (46\% versus 4\%; $p=0.0001$ ). Eight (33\%) eyes in the trabeculectomy group developed a visually significant cataract, and five (19\%) eyes developed medically uncontrolled IOP necessitating a need for trabeculectomy. At the end of the 24-month follow-up period, no eyes (0\%) in this group had medically uncontrolled IOP. ${ }^{2}$ Complications reported in the trabeculectomy group are presented in Table $1 .^{2}$

Dada et al. presented their study to assess the outcome of CLE in patients with PAC. ${ }^{3}$ In their study, CLE was performed if IOP was $\geq 25 \mathrm{mmHg}$ for $>8$ weeks following a laser peripheral iridotomy. The mean preoperative IOP was $27 \pm 1.55 \mathrm{mmHg}$, which reduced to a mean of $13.2 \pm 1.12 \mathrm{mmHg}$ at 12 months $(p<0.0001)$. Angle opening distance (AOD) at $500 \mu \mathrm{m}$ increased from baseline values measured at 0 degrees (from $0.104 \pm 0.015 \mathrm{~mm}$ to $0.31 \pm 0.013 \mathrm{~mm}$ ) and at 180 degrees (from $0.202 \pm 0.008 \mathrm{~mm}$ to $0.412 \pm 0.012 \mathrm{~mm})$. There was also improvement in trabecular-iris angle at 0 degrees from $9.3 \pm 3.2$ degrees to $32.7 \pm 5.6$ degrees, and at 180 degrees from $9.12 \pm 3.2$ degrees to $31.7 \pm 5.6$ degrees, postoperatively. Preoperatively, all patients were on 1-3 glaucoma medications. One week postoperatively, $13.6 \%$ of patients had IOP $>21 \mathrm{mmHg}$ and were re-started on one glaucoma medication. This group of patients maintained IOP at $<18 \mathrm{mmHg}$. Therefore, taking this IOP level as an absolute success, 86.4\% of patients (38 eyes) achieved this level, and $13.6 \%$ of patients (six eyes) attained qualified success. ${ }^{3}$

Man et al., in their study, compared CLE with trabeculectomy in patients with PACG. ${ }^{4}$ CLE was performed in 26 eyes, and 24 eyes underwent glaucoma filtering surgery. There was a significant reduction in mean IOP from $23.5 \pm$ $4.0 \mathrm{mmHg}$ to $14.8 \pm 3.0 \mathrm{mmHg}(p<0.001)$ by phacoemulsification alone. In the trabeculectomy group, IOP lowered from $24.1 \pm 4.4 \mathrm{mmHg}$ to $15.2 \pm$ $4.2 \mathrm{mmHg}$, postoperatively. The mean extent of synechial closure significantly reduced from $272.3 \pm 57.3$ degreesto $253.3 \pm 70.5$ degrees $(p=0.007)$ by phacoemulsification, and in the trabeculectomy group, it only reduced from $285.0 \pm 64.6$ degrees to $283.1 \pm 55.5$ degrees $(p=0.32)$. AOD at $500 \mu \mathrm{m}$ increased from $220.3 \pm 93.8 \mu \mathrm{m}$ to $388.9 \pm 134.1 \mu \mathrm{m}$ ( $\mathrm{p}<0.001)$ by $\mathrm{CLE}$, but decreased from $220.9 \pm 79.8 \mu \mathrm{m}$ to $214.5 \pm 70.2 \mu \mathrm{m}(\mathrm{p}=0.11)$ following trabeculectomy. The mean anterior chamber depth increased in the CLE group from 1,983.8 $\pm 176.8 \mu \mathrm{m}$ to $3,335 \pm 174.2 \mu \mathrm{m}(\mathrm{p}<0.001)$. However, there was a decrease from $2,000.2 \pm 214.5 \mu \mathrm{m}$ to $1,975.8 \pm 218.2 \mu \mathrm{m}(\mathrm{p}=0.001)$ in the glaucoma filtering surgery group. The authors concluded that, compared with trabeculectomy, CLE resulted in a significant reduction in synechial angle closure and an increase in anterior chamber angle width and anterior chamber depth in PACG eyes without cataract. ${ }^{4}$ 
Table 2: Summary of various studies on clear lens extraction

\begin{tabular}{|c|c|c|c|c|c|c|}
\hline Study & Year & Region & CLE (n) & $\begin{array}{l}\text { Other modes of } \\
\text { treatment }(n)\end{array}$ & $\begin{array}{l}\text { Mean preoperative IOP } \\
(\mathrm{mmHg})\end{array}$ & $\begin{array}{l}\text { Mean postoperative IOP } \\
\text { (mmHg) }\end{array}$ \\
\hline Tham et al. ${ }^{2}$ & 2013 & Hong Kong & 26 & 24 (trab) & $\begin{array}{l}24.1 \pm 4.1 \text { (CLE group); } \\
24.8 \pm 3.4 \text { (trab group) }\end{array}$ & $\begin{array}{l}15.9 \pm 3.9 \text { (CLE group); } \\
15.8 \pm 4.3 \text { (trab group) }\end{array}$ \\
\hline Dada et al. ${ }^{3}$ & 2015 & India & 44 & - & $27 \pm 1.55$ & $13.2 \pm 1.12$ \\
\hline Man et al. ${ }^{4}$ & 2015 & Hong Kong & 26 & 24 (trab) & $\begin{array}{l}23.5 \pm 4.0 \text { (CLE group); } \\
24.1 \pm 4.4 \text { (trab group) }\end{array}$ & $\begin{array}{l}14.8 \pm 3.0 \text { (CLE group); } \\
15.2 \pm 4.2 \text { (trab group) }\end{array}$ \\
\hline EAGLE study ${ }^{1}$ & 2009-2016 & Multicenter & 208 & 211 (standard care) & $\begin{array}{l}\text { 24-33 (CLE group); } \\
\text { 26-33 (standard care group) }\end{array}$ & $\begin{array}{l}16.6 \text { (CLE group); } \\
17.9 \text { (standard care group) }\end{array}$ \\
\hline Rozegnat-Madej et al. ${ }^{5}$ & 2017 & Poland & 6 & $\begin{array}{l}108 \text { (cataract } \\
\text { extraction) }\end{array}$ & & \\
\hline
\end{tabular}

$C L E=$ clear lens extraction; $I O P=$ intraocular pressure $;$ trab = trabeculectomy.

The results of the EAGLE study were published in 2016. ${ }^{1}$ Visual acuity was better in the CLE group compared with the standard care group by three Early Treatment Diabetic Retinopathy Study (ETDRS) letters. Mean IOP was $1 \mathrm{mmHg}$ lower in the CLE group compared with the standard care group at 3 years. In the CLE group, 21\% of participants received further treatment; compared with $61 \%$ in the standard care group, who were prescribed at least one glaucoma medication. The CLE group reported less frequent need for further surgeries compared with the standard care group (1 versus 24 operations). There was no significant overall difference in the number of individuals in either group experiencing deterioration of visual field (24 in CLE versus 31 in the standard care group; odds ratio $0.77,95 \%$ confidence interval [CI] 0.38-1.55). ${ }^{1}$

Rozegnat-Madej et al. reported their study of phacoemulsification and intraocular lens implantation in eyes with PAC/PACG. ${ }^{5}$ Out of the 114 eyes included in the study, six were clear lenses. However, since the study does not describe those patients separately from patients with cataracts, no conclusion can be drawn from the report. ${ }^{5}$

A summary of the outcomes in terms of IOP control, seen in the various studies mentioned above, is presented in Table 2. Based on the positive results seen in these studies, CLE is being advocated by its proponents as a first-line treatment option for PAC and PACG. ${ }^{27}$

\section{Cost-effectiveness and patient quality-adjusted life years}

One of the co-primary endpoints of the EAGLE study was to assess the incremental cost-effectiveness ratio (ICER) per quality-adjusted life year (QALY) gained after 36 months of treatment. ${ }^{1}$ The mean health service costs were higher in patients randomized to CLE, compared with standard care: $£ 2,467$ versus $£ 1,486$. The mean adjusted QALYs were also higher with early lens extraction: 2.602 versus 2.533 . The ICER for lens extraction versus standard care was $£ 14,284$ per QALY gained at 3 years. Using certain modeling methods, it was calculated that ICER would drop to $£ 7,090$ per QALY gained by 5 years, and expected to be cost-saving by 10 years. ${ }^{28}$ CLE would reduce the need for glaucoma therapy while maintaining good visual acuity. These factors would improve the quality of life in patients with glaucoma. ${ }^{6}$

\section{Intraoperative mechanisms}

Several mechanisms have been proposed through which CLE reduces IOP. Deepening of anterior chamber with viscoelastic and hydrostatic pressure during surgery could mechanically strip open some pre-existing peripheral anterior synechiae, especially those with weak adherence to the trabecular meshwork. The forceful irrigation during phacoemulsification could also flush open the trabecular meshwork. ${ }^{29}$ Cataract extraction deepens the anterior chamber, opens the angle, and increases aqueous outflow facility. ${ }^{13}$ Increased release of prostaglandins, interleukins, and tumor necrosis factors during the perioperative period is also suspected to improve aqueous outflow. ${ }^{4,30,31}$ Handa et al. have suggested cataract extraction could increase aqueous outflow through the uveoscleral pathway by possible alterations in the blood-aqueous barrier. ${ }^{32}$ In-the-bag IOL implantation apparently stretches the zonules to create traction on the trabecular meshwork. This could open up the trabecular spaces and facilitate aqueous outflow. ${ }^{4,33} \mathrm{~A} 4.0 \mathrm{~mm}$ capsulorhexis produces more traction on the zonules compared with a $6.0 \mathrm{~mm}$ rhexis. ${ }^{11} \mathrm{CLE}$ also resolves pupillary block and attenuates plateau iris mechanism by repositioning the ciliary processes in a more posterior location..$^{10,24}$ Other postulated mechanisms include partial ciliary body detachment and alterations in the iris root. ${ }^{33-35}$

One probable disadvantage of glaucoma filtering surgery is reduced aqueous flow through the trabecular meshwork causing under-perfusion and leading to degenerative changes similar to those seen in chronic open-angle glaucoma. Conversely, phacoemulsification induces positive changes in the anterior chamber configuration without inducing trabecular meshwork hypoperfusion. ${ }^{36}$

Cataract surgery in the past and CLE now appear to provide patient-reported and clinical advantages, which support the introduction of this concept as a first-line treatment for individuals with PACD.

\section{Negative aspects of studies on clear lens extraction}

PACD is not a single disease entity; rather, it is a continuum of different stages. Thus, each individual can be unique in terms of disease stage, and the management profile has to be tailored based on that particular stage. The International Society of Geographic and Epidemiologic Ophthalmology has classified PACD into primary angle-closure suspect (PACS), primary angle-closure (PAC), and PACG. ${ }^{37}$ Thomas et al. have described the role of lens extraction in the management of each stage. In PACS, the authors advocate prophylactic peripheral iridotomy due to the relative safety of the procedure without resorting to CLE. However, in PACG, CLE has shown good results in terms of IOP lowering, reduced need for medications, and 
a decrease in peripheral anterior synechiae. In some patients, adequate lowering of IOP may not occur despite the opening of the angles; these eyes may require glaucoma filtering surgery in the future. However, filtering surgery in such eyes will have to be deferred by 6 months due to postoperative inflammation following CLE. In sensitive individuals, the compromised optic disc may not be able to tolerate the postoperative IOP spike. ${ }^{37}$

Freidman and Vedula have reviewed a number of databases to assess the effectiveness of lens extraction in PACD. The authors did not find any evidence to support lens extraction as a treatment for chronic PACG. ${ }^{38}$ Similarly, Lam et al. have advocated further debate regarding the risk-benefit ratio of phacoemulsification in eyes with good visual acuity (20/40 or better) following the abortion of an acute attack or without cataract. ${ }^{39}$

\section{Randomization of patients}

In the EAGLE study, 805 individuals were assessed for inclusion. However, 386 patients were found to be ineligible or refused to participate. Out of the 419 patients included in the study, 208 patients underwent CLE. Exclusion of nearly half of the patients during the recruitment stage itself may suggest the potential for sampling bias. A large group of patients were either satisfied with their visual status or were reluctant to undergo CLE. Therefore, the study effectively recruited a substantial number of patients with proactive characteristics who were probably inclined towards better visual outcome and quality of life following CLE. ${ }^{1,40}$ It is also not clear how many patients had lens-related factors like lens vault or intumescence.

Questionnaires, such as the European Quality of Life-5 Dimensions questionnaire (EQ-5D) and the 25-item National Eye Institute Visual Function questionnaire (NEI-VFO-25), are based on the patient's perception of change and may have yet another potential bias. Patients, especially those who are unable to read, are often unable to appreciate the potential complications of surgical procedures. This may give rise to erroneous and biased results in such questionnaire assessments. ${ }^{40}$ In the EAGLE study, eyes with PAC and PACG were treated on the assumption that the two study groups would respond to the treatment modalities in a similar fashion. However, it is presumptive how generalizable the study's findings would be to other patients. ${ }^{41}$

\section{Preoperative management}

In the EAGLE study, intervention was performed after 60 days of randomization. ' In Dada's study, CLE was undertaken if IOP remained $\geq 25$ mmHg for $>8$ weeks after peripheral iridotomy. ${ }^{3}$ In the EAGLE study, the choice of therapy, such as medications, laser, or surgery, was based on "local protocols". Since details of these protocols are not available, it is not possible to analyze the influence of those factors in the study results. ${ }^{42}$

In Dada's study, preoperatively $13.6 \%$ of patients were on one medication, $70.5 \%$ were on two, and $15.9 \%$ were on three medications. Thus, a majority of patients were on a maximum of two medications. Patients underwent surgery 8 weeks after diagnosis. This period appears to be too short to provide meaningful alteration in the treatment regime. In the author's own experience, patients often require change or addition of topical glaucoma medications to discover the most appropriate drug effective in a particular patient. There have been instances when patients were uncontrolled by up to four different types of eyedrops, but then a single drug was ultimately found, which alone was sufficient to keep IOP within the target range. However, time and effort are required in order to find that particular medication. It is not possible to reach such conclusions within 6-8 weeks. In Dada's study nearly $14 \%$ of patients were on only one glaucoma medication. Would the results be different if medications were added or changed?

In the EAGLE study, preoperative IOP was $\geq 30 \mathrm{mmHg}$, but no peripheral iridotomy was performed to lower IOP. The longer the duration of elevated IOP, the more the damage to intraocular structures. Azuara-Blanco et al. performed an experimental study whereby IOP was artificially raised for a short period. This caused a significant increase in cup volume and cup area. ${ }^{43}$ Roberts et al. mentioned that IOP elevation for $\geq 3$ days lowers the central endothelial count significantly. Conversely, raised IOP for only a few hours or up to 2 days may not affect the cell count. ${ }^{36}$ Therefore, not performing a procedure that is safe, easy and effective, such as peripheral iridotomy or iridoplasty, and waiting 4-8 weeks to perform a CLE, appears to be improper.

\section{Peripheral anterior synechiae}

The presence of peripheral anterior synechiae is regarded as an indication for CLE, as peripheral iridotomy may not be effective. Nonetheless, in a study by Lai et al., some eyes with only 90 degrees of angle-closure following cataract extraction required glaucoma medications, while others with $>270$ degrees of angle-closure did not..$^{33}$ Chen et al. have also mentioned that IOP reduction following lens extraction does not seem to be related to the extent of peripheral anterior synechiae prior to surgery, but is more related to angle width. ${ }^{24}$ Gonioscopy, conducted before lens extraction, may overestimate the degree of synechial angle closure. The procedure is subjective, semi-quantitative and may not be adequate to determine the severity of ACG.

Huang et al., using aqueous angiography, demonstrated segmental, pulsatile, and dynamic angiographic aqueous outflow through the anterior chamber. Positive flow was suddenly observed in regions previously without any angiographic signal and conversely the signal would disappear in regions where it was initially documented. ${ }^{44}$ Loewen et al., using fluorescein canalograms, found significantly faster flow in inferonasal and superonasal quadrants compared with superotemporal and inferotemporal locations $(p<0.05) .{ }^{45}$ Therefore, it can be argued that the position, rather than the extent of iridotrabecular contact and peripheral anterior synechiae, may be significantly important factors in the development of elevated IOP. These findings may have clinical implications when considering relationships between angle closure and IOP elevation.

\section{Refractive outcomes}

Patients with glaucoma have an increased propensity to show postoperative refractive surprises compared with the healthy population. In a study conducted at the University of Colorado, USA, significantly higher frequency of both small and large magnitude of refractive surprises were found in patients with glaucoma, as compared with patients without glaucoma. ${ }^{46}$ The postoperative prediction of the effective lens position significantly relies on anterior chamber depth estimates, and is considered a major limiting factor in modern IOL power calculations. ${ }^{47}$ Other factors, such as corneal curvature, axial length and lens vault, are also variable in patients with PACD or not included in formulas to calculate IOL power. ${ }^{30,48}$ 
In the EAGLE study, visual acuity was better in the CLE group compared with the standard care group by just three ETDRS letters. The authors of the EAGLE study pointed out that "while this magnitude of change is unlikely to be clinically important, it points to the overall improvement in visual function associated with CLE." ${ }^{\prime \prime}$ It is possible that the so-called clear lenses in the EAGLE study had some degree of nuclear sclerosis and developed a "refractive surgery effect" following CLE. ${ }^{49}$ Day et al., in their analysis of the EAGLE study, agreed that refractive predictability was sub-optimal. ${ }^{50}$ In the study by Lam et al., postoperative visual gain was persistently better in the phacoemulsification group compared with the standard care group, but not statistically significant. ${ }^{39}$ LOSS of accommodation following CLE in a relatively young population should also be considered.

\section{Perioperative complications}

Laser peripheral iridotomy and iridoplasty are effective office procedures and can be performed immediately on assessing the need for intervention. ${ }^{51,52}$ Conversely, lens extraction has to be planned and patients are often skeptical of surgical options. In developing countries, patients often need to discuss the situation with families, as well as arrange finances for surgery. This requires an intervening period, which may last for months at times. Patients occasionally travel long distances to reach centers equipped for laser/surgical procedures. To delay a simple office procedure such as peripheral iridotomy may prove disastrous, as the patient may develop an AAC attack during the waiting period.

Immediately prior to surgery, the pupil has to be dilated. The risk of developing an acute AAC attack due to pupillary dilatation has to be kept in mind. ${ }^{53}$ PAC eyes have thick, large lenses with shallow anterior chambers. During surgery, there is less room for the surgeon to maneuver the bulky nucleus in the confined space. This may force the surgeon to apply phaco power near the corneal endothelium. ${ }^{10}$ Studies on cataract extraction in PACD eyes have reported postoperative decreased corneal endothelial cell counts and corneal decompensation. ${ }^{33,4,54}$ Individuals with a previous history of AAC may have decreased endothelial cell density by around $33 \%$ from baseline and are vulnerable for corneal decompensation.2.233 Zonular instability may also be a factor in these patients. ${ }^{5}$ Eyes with PACD may have atonic or atrophied irises, making pupillary dilatation difficult. This may also occur following long-term use of pilocarpine, or in the presence of significant posterior synechiae. In these situations, pupillary stretching by hooks, rings, or other maneuvers would be required. This increases the risk of iris trauma, hyphema, and chronic inflammation. The atonic iris may also cause intraoperative floppy iris syndrome and aggravate postoperative uveitis.

In the EAGLE study, complications encountered in the CLE group were as follows: intraoperative-posterior capsule tear $(n=2 ; 1 \%)$, iris prolapse $(n=2 ; 1 \%)$, vitreous loss $(n=1 ; 0.5 \%)$; postoperative-flat anterior chamber $(n=2 ; 1 \%)$, malignant glaucoma $(n=1 ; 0.5 \%)$, corneal edema $(n=1 ; 0.5 \%)$, macularedema $(n=5 ; 2.4 \%)$, spikein IOP $(n=2 ; 1 \%)$, postoperative inflammation $(n=5 ; 2.4 \%)$, macular hole $(n=1 ; 0.5 \%)$, and systemic events $(n=2 ; 1 \%) .^{1}$ In the study by Tham et al., one patient (4\%) had zonular dehiscence requiring implantation of a capsular tension ring in the CLE group. On the other hand, complications in the trabeculectomy group were managed successfully in all patients. ${ }^{2}$ IOP spikes in the immediate postoperative period may occur in $9-60 \%$ of eyes. 1029,37 This can cause further optic nerve damage and even lead to postoperative snuff-out syndrome. In all CLE studies, nearly $3 \%$ of patients had some degree of intraoperative complications despite being operated by experienced surgeons. The outcome of operating such technically difficult cases by less experienced surgeons may prove catastrophic.

Postoperative endophthalmitis rates have shown a rising tendency ever since small-incision sutureless cataract surgery became popular. In Asian countries, the incidence of postoperative endophthalmitis varies from $0.076-0.359 \% .{ }^{55}$ Looking at the agrarian lifestyle and under-developed status in certain regions of the world, CLE has the potential to significantly increase the risk of postoperative endophthalmitis. Cataract surgery may lead to posterior capsule opacification. It is reported that capsulotomy for posterior capsule opacification may cause an early, transient rise ( $>5 \mathrm{mmHg}$ ) of IOP in 10-40\% of all individuals and in one-fifth of all patients with glaucoma. ${ }^{30}$ Thus, there is increased risk of wipe-out syndrome in these patients.

Any postoperative complications can unnecessarily create visual problems where none existed before CLE. These can cause psychological distress and financial burden on patients, as they have to travel frequently to the hospitals for follow-up. ${ }^{56}$ Any surgical procedure also requires control of co-morbid conditions such as diabetes mellitus, systemic hypertension, infections, and inflammations. This is not a significant factor when performing peripheral iridotomy.

\section{Regression to the mean}

The significantly lowered IOP mentioned in many reports is often ascribed to the phenomenon of "regression to the mean". It implies that the statistically lowered postoperative IOP is an exaggerated effect on the relatively high preoperative IOP in the inclusion criteria. This makes the lowering of IOP from a very high level to lower levels statistically significant. ${ }^{33}$ Chen et al. have noted that "higher IOP is the single most significant factor associated with a greater IOP drop after phacoemulsification" ${ }^{24}$ Similar results have also been reported by other authors. ${ }^{1149,57-59}$ Baseline IOP in the EAGLE study was $\geq 30 \mathrm{mmHg}$ and in Dada's study was $\geq 25 \mathrm{mmHg}$. ${ }^{1.3}$ Therefore, the significant postoperative lowering in IOP seen in these studies could be attributed to regression to the mean.

\section{Intraocular pressure}

In the EAGLE study, preoperative IOP was $\geq 30 \mathrm{mmHg}$. Therefore, questions remain whether CLE would be useful if IOP is lower. ${ }^{60}$ The mean postoperative IOP in the EAGLE study was $1 \mathrm{mmHg}$ lower in the CLE group at 3-year follow-up. Clinically, this difference is small and statistically insignificant. ' In Dada's study, good IOP control was noted for 12 months after CLE. ${ }^{3}$ Whether this effect would be sustained beyond this period is not known. Vizzeri and Weinreb have opined that lens extraction alone may not be sufficient for long-term IOP control. ${ }^{59}$ Studies show IOP reduction in eyes with PACG ranging from 2-12 mmHg. ${ }^{28}$ However, the lowering of IOP is also dependent on trabecular and post-trabecular mechanisms. Dysfunction in these regions may not provide adequate lowering of IOP by CLE alone. ${ }^{7.37}$ The effect of CLE on diurnal fluctuation of IOP is also questionable. ${ }^{37}$ Some suggest this can be better achieved by glaucoma filtering surgery. ${ }^{59}$

\section{Visual field changes}

In the EAGLE study, the number of individuals in both the CLE and standard care groups with visual field deterioration (24 in CLE versus 31 in standard care groups) did not differ significantly (odds ratio $0.77,95 \% \mathrm{Cl} 0.392-1.511$ ). 
However, the number of glaucoma surgeries performed subsequently in the two groups have significant differences (one in the CLE group versus 24 in standard care). This likely indicates that although there was no glaucomatous progression observed in both groups, surgery was performed ostensibly to reduce IOP or the number of medications. This is not explained in detail in the study. Also, long-term effects on visual fields are not available in the EAGLE study. ${ }^{40}$

\section{Cost-effectiveness}

The EAGLE study showed that CLE may have better ICER compared with standard care. CLE may prove to be more economically friendly by avoiding life-long expenditure on glaucoma medications. There are two objections to this hypothesis. Firstly, it is not inevitable that every patient with glaucoma will develop cataract. To quote Thomas et al., "the disease may outlive the patient." ${ }^{\prime 7}$ For example, if we use keratoconus as an analogy; patients with keratoconus may develop acute hydrops, and those with progressive keratoconus or hydrops may require corneal transplants. However, doing keratoplasty (which has excellent prognosis in keratoconus) in all patients, based on the assumption that they all will develop hydrops in the future, is unfathomable. Similarly, removing the lenses of all patients with PACD because they may develop cataracts, is a seriously flawed argument. Secondly, in some countries, glaucoma medications are provided by government hospitals through a tax-paying or social-service mechanism. However, most of these countries require the patient to pay for surgery, leading to the incremental cost of treatment.

Indices such as EQ-5D and Glaucoma Utility Index are largely dependent on visual status. Similarly, bilateral visual status forms the cornerstone of ICER.
The positive results in these parameters seen in the EAGLE study are likely due to improved contrast sensitivity and not angle-closure status. ${ }^{42}$

The financial advantage mentioned in the EAGLE study is specific to the UK and may not be applicable in countries where insurance companies may refuse CLE due to a lack of visual complaints. In the EAGLE study, CLE would presumably be cost-effective only after 10 years of surgery. ${ }^{28}$ The risks and benefits of surgery during such a period have to be taken into consideration.

\section{Conclusion}

CLE is being advocated as a method to primarily treat PACD without opting for other standard care modalities. The advocates for this procedure claim CLE has the advantage of treating the cause of PACD, i.e., the lens, thus the method is curative to some degree. However, like many surgical procedures, CLE is associated with increased risk of intra- and postoperative complications and other social factors compared with standard care practices. It is essential to individualize each patient and management tempered by various factors, not just IOP control. Any new method for management of PACD needs to be proven beneficial compared with standard care guidelines being practiced currently. This review highlights the positive and negative aspects of the studies performed so far on the role of CLE in this disease process.

CLE remains controversial as the risks involved in operating a large volume of patients with no visual complaints opens a potential medico-legal minefield. How the method will fare in terms of generalized acceptance in the future is a matter of conjecture. $\square$
1. Azuara-Blanco A, Burr J, Ramsay C, et al. Effectiveness of early lens extraction for the treatment of primary angle-closure glaucoma (EAGLE): a randomized controlled trial. Lancet. 2016;388:1389-97.

2. Tham CC, Kwong YY, Baig N, et al. Phacoemulsification versus trabeculectomy in medically uncontrolled chronic angle closure glaucoma without cataract. Ophthalmology. 2013;120:62-7.

3. Dada T, Rathi A, Angmo D, et al. Clinical outcomes of clear lens extraction in eyes with primary angle closure. J Cataract Refract Surg 2015:41:1470-7.

4. Man X, Chan NCY, Baig N, et al. Anatomical effects of clear lens extraction by phacoemulsification versus trabeculectomy on anterior chamber drainage angle in primary angle-closure glaucoma (PACG) patients. Graefes Arch Clin Exp Ophthalmol. 2015;253:773-8.

5. Rozegnat-Madej A, Wilkos-Kuc A, Wlaz A, Zarnowski T. Evaluation of efficacy of lens extraction for intraocular pressure reduction in eyes with primary angle-closure glaucoma and primary angle-closure. Ophthalmol J. 2017;2:6-12.

6. Singh $\mathrm{K}$, Dangda S. Role of lens extraction in glaucoma - a review. Delhi J Ophthalmol. 2016;26:235-40.

7. Krishnadas R. Current management options in primary angle-closure disease. Indian J Ophthalmol. 2019;67:321-3.

8. Lowe RF. Aetiology of the anatomical basis for primary angle closure glaucoma. Br J Ophthalmol. 1970;54:161-9.

9. Poley BJ, Lindstrom RL, Samuelson TW, et al. Intraocular pressure reduction after phacoemulsification with intraocular lens implantation in glaucomatous and nonglaucomatous eyes; evaluation of a causal relationship between the natural lens and open-angle glaucoma. J Cataract Refract Surg. 2009;35:1946-55

10. Tarongoy $\mathrm{P}, \mathrm{Ho} \mathrm{CL}$, Walton DS. Angle-closure glaucoma: the role of the lens in the pathogenesis, prevention and treatment. surv Ophthalmol. 2009:54:211-25.

11. Shin HC, Subrayan V, Tajunisah I. Changes in anterior chamber depth and intraocular pressure after phacoemulsification in eyes with occludable angles. J Cataract Refract Surg. 2010;36:1289-95

12. Weinreb RN, Aung T, Medeiros FA. The pathophysiology and treatment of glaucoma. JAMA. 2014;311:1901-11

13. Vasile $\mathrm{P}$, Catalina $\mathrm{C}$. The role of clear lens extraction in angle closure glaucoma. Rom J Ophthalmol. 2017;61:244-8.

14. Moghimi $\mathrm{S}$, Hashemian $\mathrm{H}$, Chen $\mathrm{R}$, et al. Early phacoemulsification in patients with acute primary angle-closure. J Curr Ophthalmol. 2015:27:70-5.

15. Lee HS, Park JW, Park SW. Factors affecting refractive outcome after cataract surgery in patients with history of acute primary angle-closure. Jpn J Ophthalmol. 2014;58:33-9.
16. Guyton JS. Choice of operation in eyes with primary glaucoma and cataracts. Trans Am Acad Ophthalmol Otolaryngol. 1945;49:216-24.

17. Bigger $F, B e c k e r ~ B$. Cataracts and primary open-angle glaucoma: the effects of uncomplicated cataract extraction on glaucoma control. Trans Am Acad Ophthalmol Otolaryngol. 1971;75:260-72.

18. Civerchia LL, Balent A. Intraocular lens implantation in acute angle closure glaucoma associated with cataract. Am Intra-Ocular Implant SOC J. 1985:11:171-3.

19. Wishart PK, Atkinson PL. Extracapsular cataract extraction and posterior chamber lens implantation in patients with primary angle-closure glaucoma: effect on intraocular pressure contro Eye (Lond). 1989;3:706-12.

20. Guning FP, Greve EL. Lens extraction for uncontrolled angle-closure glaucoma: Iong term follow-up. J Cataract Refract Surg. 1998:24:1347-56.

21. Hata $\mathrm{H}$, Yamane $\mathrm{S}$, Hata $\mathrm{S}$, et al. Preliminary outcomes of primary phacoemulsification plus intraocular lens implantation for primary angle-closure glaucoma. J Med Invest. 2008;55:287-91.

22. Jacobi PC, Dietlin TS, Luke C, et al. Primary phacoemulsification and intraocular lens implantation for acute angle-closure glaucoma. Ophthalmology. 2002;109:1597-603.

23. Tham CC, Kwong YY, Leung DY, et al. Phacoemulsification versus combined phacotrabeculectomy in medically uncontrolled chronic angle closure glaucoma with cataracts. Ophthalmology 2009:116:725-31.

24. Chen PP, Lin SC, Junk AK, et al. The effect of phacoemulsification on intraocular pressure in glaucoma patients. Ophthalmology. 2015;122:1294-307.

25. Walland $\mathrm{M}$, Thomas R. Role of clear lens extraction in adult angle closure disease: a review. Clin Exp Ophthalmol. 2011;39:61-4.

26. Azura-Blanco A, Burr JM, Cochran C, et al. The effectiveness of early lens extraction with intraocular lens implantation for the treatment of primary angle-closure glaucoma (EAGLE): study protocol for a randomized controlled trial. Trials. 2011;12:133

27. Lusthaus J, Goldberg I. Current management of glaucoma Med J Aust. 2019;210:180-7.

28. Javanbakht M, Azura-Blanco A, Burr JM, et al. Early lens extraction with intraocular lens implantation for the treatment of primary angle closure glaucoma: an economic evaluation based on data from the EAGLE trial. BMJ Open. 2017;6:e013254.

29. Trikha $S$, Perera $S A$, Husain $R$, et al. The role of lens extraction in the current management of primary angle-closure glaucoma. Curr Opin Ophthalmol. 2015;26:128-34.

30. Eid TM. Primary lens extraction for glaucoma management: a review article. Saudi J Ophthalmol. 2011;25:337-45.
31. Shrivastava $A$, Singh $K$. The effect of cataract extraction on intraocular pressure. Curr Opin Ophthalmol. 2010;21:118-22.

32. Handa J, Henry JC, Krupin T, et al. Extracapsular cataract extraction with posterior chamber lens implantation in patients with glaucoma. Arch Ophthalmol. 1987;105:765-9.

33. Lai JSM, Tham CCY, Chan JCH. The clinical outcomes of cataract extraction by phacoemulsification in eyes with primary angle closure glaucoma (PACG) and co-existing cataract (a prospective series). J Glaucoma. 2006:15:47-52

34. Teekhasaenee C, Ritch R. Combined phacoemulsification and goniosynechialysis for uncontrolled chronic angle-closure glaucoma after acute angle-closure glaucoma. Ophthalmology. 1999;106:669-75.

35. Shams PN, Foster PJ. Clinical outcomes after lens extraction for visually significant cataract in eyes with primary angle closure. J Glaucoma. 2012;21:545-50

36. Roberts TV, Francis IC, Lertusumitkul S, et al. Primary phacoemulsification for uncontrolled angle-closure glaucoma. J Cataract Refract Surg. 2000;26:1012-6.

37. Thomas R, Walland MJ, Parikh RS. Clear lens extraction in angle closure glaucoma. Curr Opin Ophthalmol. 2011;22:110-4.

38. Friedman D, Vedula SS. Lens extraction for chronic angle-closure glaucoma. Cochrane Database Syst Rev. 2006:CD005555.

39. Lam DSC, Leung DYL, Tham CCY, et al. Randomized trial of early phacoemulsification versus peripheral iridotomy to prevent intraocular pressure rise after acute primary angle closure. Ophthalmology. 2008;15:1134-40.

40. Chan PP, Tham CC. Commentary on effectiveness of early lens extraction for the treatment of primary angle-closure glaucoma (EAGLE). Ann Eye SCi. 2017;2:21.

41. Traverso CE. Clear lens extraction as a treatment for primary angle-closure. Lancet. 2016:388:1352-4.

42. Sihota R. Treatment of primary angle-closure glaucoma: does early lens extraction help? Natl Med J India. 2017;30:78-9.

43. Azuara-Blanco A, Harris A, Cantor L, et al. Effects of short term increase of intraocular pressure on optic disc cupping. Br J Ophthalmol. 1998;82:880-3

44. Huang $A S$, $L i M$, Yang $D$, et al. Aqueous angiography in living non-human primates show segmental, pulsatile and dynamic angiographic aqueous humor outflow. Ophthalmology. 2017; 124:793-803.

45. Loewen RT, Brown EN, Roy P, et al. Regionally discrete aqueous humor outflow quantification using fluorescein canalograms. PLOS ONE. 2016;11:e0151754

46. Manoharan N, Patnaik JL, Bonnell LN, et al. Refractive outcomes of phacoemulsification cataract surgery in glaucoma patients. 
J Cataract Refract Surg. 2018;44:348-54.

47. Jeong J, Song H, Lee JK, et al. The effect of ocular biometric factors on the accuracy of various intraocular lens power calculation formulas. BMC Ophthalmology. 2017;17:62.

48. Joo J, Whang WJ, Oh TH, et al. Accuracy of intraocular lens power calculation formulas in primary angle closure glaucoma. Korean J Ophthalmol. 2011;25:375-9.

49. Traverso CE, Cutolo CA. The effects of phacoemulsification and intraocular lens implantation on anatomical and functional parameters in patients with primary angle closure: a prospective study. Trans Am Ophthalmol Soc. 2017;115:T7.

50. Day AC, Cooper D, Burr J, et al. Clear lens extraction for the management of primary angle closure glaucoma: surgical technique and refractive outcomes in the EAGLE cohort. Br J Ophthalmol. 2018;102:1858-62.
51. Zebardast N, Kavitha S, Krishnamurthy P, et al. Changes in anterior segment morphology and predictors of angle widening after laser iridotomy in South Indian eyes. Ophthalmology. 2016;123:2519-26.

52. Leong JCY, O'Connor J, Ang GS, et al. Anterior segment optical coherence tomography changes to the anterior chamber angle in the short-term following laser peripheral iridoplasty. the short-term following laser peripher
$\mathrm{J}$ Curr Glaucoma Pract. 2014;8:1-6.

53. Tanner L, Gazzard G, Nolen WP, et al. Has the EAGLE landed for the use of clear lens extraction in angle-closure glaucoma? And how should primary angle-closure suspects be treated? Eye (Lond). 2020;34:40-50

54. Kubota T, Togiuri I, Onizuka N, et al. Phacoemulsification and intraocular lens implantation for angle closure glaucoma after the relief of pupillary block. Ophthalmologica. 2003;217:325-8.
55. Choi JA, Chung SK. Postoperative endophthalmitis following cataract surgery in Asia. ISRN Ophthalmol. 2011:917265.

56. Shi K, You Z, Wang C. Clear lens extraction for early angle-closure glaucoma: revolution or overtreatment? Med Princ Pract. 2017;26:297-8.

57. Wedrich A, Menapace R, Radax U, et al. Long-term results of combined trabeculectomy and small incision cataract surgery. J Cataract Refract Surg. 1995;21:49-54

58. Liu CJ, Cheng CY, Ko YC, et al. Determinants of long-term intraocular pressure after phacoemulsification in primary angle closure glaucoma. I Glaucoma. 2011;20:566-70.

59. Vizzeri G, Weinreb RN. Cataract surgery and glaucoma. Curr Opin Ophthalmol. 2010;21:20-4.

60. Ling JD, Bell NP. Role of cataract surgery in the management of glaucoma. Int Ophthalmol Clin. 2018;58:87-100. 